\title{
MENSCHLICHE MYIASIS: KLINISCHER FALL
}

\section{ORIGINALER ARTIKEL}

SILVA, Agnaldo Plácido da ${ }^{1}$, PLÁCIDO, Eloá Jessica Mendes dos Santos², MORAES, Walber Breno de Souza ${ }^{3}$

SILVA, Agnaldo Plácido da. PLÁcIDO, Eloá Jessica Mendes dos Santos. MORAES, Walber Breno de Souza. Menschliche Myiasis: Klinischer Fall. Revista Científica Multidisciplinar Núcleo do Conhecimento. Jahrgang 05, Ed. 08, Vol. 10, S. 39-46. August 2020.

ISSN: 2448-0959,

Zugriffsverbindung: https://www.nucleodoconhecimento.com.br/gesundheit/humanmy iasis, DOI: 10.32749/nucleodoconhecimento.com.br/gesundheit/humanmyiasis

\section{ZUSAMMENFASSUNG}

Myiasis gilt als Dermatozoonosebefall, der durch den Befall von Dipteranlarven in Geweben oder Organen verursacht wird, die ihre Eier in Menschen oder Tieren legen, die sich für einen bestimmten Zeitraum von lebenden oder toten Geweben des Wirts ernähren, das Auftreten von Myiasis in der Mundhöhle kann als eine seltene Sache angesehen werden. Diese Art von Krankheit betrifft am häufigsten Menschen mit niedrigem sozioökonomischen Status, immungeschwächte, bettlägerige ältere Menschen und mit psychiatrischen Störungen. Aufgrund seines großen destruktiven Potenzials, einer rechtzeitigen und wichtigen angemessenen Prävention und Behandlung, gibt es auch wenig Wissen über den Zahnarzt für die Diagnose und Behandlung einer solchen Pathologie, Aus diesem Grund berichtet die vorliegende Studie einen klinischen Fall von oraler Myiasis bei einem älteren Menschen

\footnotetext{
${ }^{1}$ Doktorand in Biomedizinischen Wissenschaften am IUNIR- Universität / Institut Italiano de Rosário Argentinien. Spezialist für Kiefer- und Gesichtschirurgie und Traumatologie von Ciodonto - Integrierte Zahnklinik. Abschluss in Zahnmedizin von ASCES - Caruaruense-Vereinigung von Universitäts Bildung. Abschluss als Biologe an der UPE - Universität Pernambuco.

${ }^{2}$ Absolvent des Physiotherapiekurses des UNINASSAU College.

${ }^{3}$ Studium der Biologie UPE- Universität Pernambuco. Master in natürlichen Ressourcen - UFCG. Professor bei UNOPAR.
}

RC: 61098

Disponível

em: https://www.nucleodoconhecimento.com.br/gesundheit/humanmyiasis 
bettlägerig mit einer Geschichte von bösartigen Kehlkopf/glottalen NeoplasieVerletzungen, die körperliche und geistige Schwäche und unzureichende Körperhygiene und Mangel an Lippenversiegelung, die zu dem Infall geführt. Die Diagnose wurde klinisch auf der Grundlage der Beobachtung von Zungenblutungen, Gewebeablösung und Vorhandensein von Larven, die zwischen dem zweiten und dritten Entwicklungsstadium waren, festgestellt. Die Behandlung wurde als Krankenhausaufenthalt des Patienten für die Debridement von nekrotischen Geweben und Entfernung von Larven und Verschreibung von Ivermectin eingeleitet. Bedridden Patienten mit körperlicher und Metallschwäche stellen eine Risikogruppe für orale Myiasis dar, und es ist von großer Bedeutung, Pflegekräfte und Familienmitglieder in Bezug auf die Zahnpflege zu führen, um diese Pathologie zu verhindern. Jedoch, das Fehlen eines Konsenses über den besten therapeutischen Ansatz für Fälle von oraler Myiasis wurde als therapeutisches Verhalten für die Krankheit die mechanische Entfernung von Larven und Institution der oralen Verwendung von Ivermectin genommen.

Stichworte: Larve, menschliche Myiasis, Fliegen, öffentliche Gesundheit.

\section{EINFÜHRUNG}

Myiasis ist definiert als eine Pathologie, bei der Fliegenlarven, die sich von lebenden oder toten Geweben ernähren, pestilent sind (VINIT; JAYAVELU und SHRUTHA, 2013), obwohl diese Parasitose seit vielen Jahren von Forschern als technischer Begriff bezeichnet wird bicheira wurde 1840 von dem ehrwürdigen F.W Hope in seiner Arbeit "On insects and their larvae occasionally found in the human body" vorgeschlagen, wo vorgeschlagen wird, den Begriff "Myiasis" zu verwenden, der sich aus den griechischen Wörtern myia (Fliege) und ase (ableitet). Krankheit) (GUIMARAES; PAPAVERO und PRADO, 1983). In der hinduistischen Literatur wurde Myiasis von der Mythologie als Bestrafung für Sünder angesehen (PARMAR; DAVESSAR et al., 2018).

RC: 61098

Disponível

em: https://www.nucleodoconhecimento.com.br/gesundheit/humanmyiasis 
Patienten mit Myiasis stellen in der Regel einige Faktoren dar, die zu einer Veranlagung für ihre Entwicklung führen, wie geistige Retardierung, psychiatrische Störungen, Senilität, Gefäßerkrankungen, Neoplasmen, Diabetes und Immundepression und chronischer Alkoholkonsum, und tritt vorzugsweise bei älteren Menschen auf. (MARQUEZ; MATTOS und NASCIMENTO, 2007) (SILVEIRA; PINHEIRO et al., 2015) Die Faktoren, die für den Beginn der Myiasis von größter Bedeutung sind, sind jedoch nach wie vor das Auftreten von exponierten Läsionen mit Exsudationen und schlechter Körper- und Mundhygiene (NASCIMENTO; OLIVEIRA et al., 2005) (TOURÉ, 1994).

Dies ist eine Situation von tödlicher Schwere für den Patienten, zusätzlich zu Schmerzen und Gewebezerstörung. Orale Myiasis kann als Zerrung der Mundhöhle, Schwellung der Mundschleimhaut, Entzündung des Zahnfleisches (RIBEIRO; PEREIRA et al., 2001). "Im Fall von Myiasis cavitaria, die die Regionen mund und Nase und Nasennebenhöhlen umfasst, kann bestätigt werden, dass es sich um eine sehr seltene Art von Myiasis handelt" (BARNAB; FERRAZ et al., 2016). Die am weitesten verbreitete Vielfalt von Fliegenarten verantwortlich für die meisten Fälle von Myiasis gehören Cochliomyia hominivorax, Chrysomya bezziana, Oestrus ovis, Magnificent Wohlfahrtia und die heimische Musca. Die Inzidenz von Infektionen ist selbst in Entwicklungsländern selten (KHAN; SABIR et al., 2018) (TOURÉ, 1994).

Die Behandlung der Myiasis besteht grundsätzlich in der manuellen Entfernung der Larven mit Hilfe einer Kürette und einer klinischen Pinzette, meist unter Narkose oder Analgesie, je nach klinischem Zustand des Patienten muss die Entfernung der Larven sorgfältig erfolgen, damit die Larven werden nicht fragmentiert und ihre Überreste bleiben an Ort und Stelle, was einen infektiösen Prozess auslöst (CAVALCANTI, 2008), obwohl es einige Produkte auf dem Markt gibt, die verwendet werden können, um die Entfernung zu erleichtern (BARNABÉ; FERRAZ et al., 2016), die Literatur berichtet mit großem Erfolg über die systemische Anwendung einer Dosis von Ivermectin, einem halbsynthetischen Makrolid-Antibiotikum, zur Behandlung schwererer Fälle mit Vorliegen einer oralen Myiasis, die sich in den

RC: 61098

Disponível

em: https://www.nucleodoconhecimento.com.br/gesundheit/humanmyiasis 
empfohlenen Dosierungen von $200 \mu \mathrm{g} / \mathrm{kg}$ als sichere Methode erwiesen. Selbst bei Dosierungen von 300 bzw. $400 \mu \mathrm{g} / \mathrm{kg}$ zeigten die behandelten Patienten bis auf leichten Juckreiz keine wesentlichen klinischen Nebenwirkungen (GEALH; FERREIRA et al., 2009) (RIBEIRO; PEREIRA et al., 2001).

Wir können Myiasis in drei verschiedene Arten einteilen: biontophage oder primäre, die dipteranLarven sind, die sich auf oder innerhalb von Wirbeltieren entwickeln und sich von den Geweben des lebenden Wirts ernähren. Unter diesen Arten finden wir Cochliomy homini vorax und Dermatobia hominis. Sekundäre Nekrobiolline sind solche, bei denen sich Dipteranlarven zu organischer Substanz entwickeln, die bereits zersetzungsmittelist (Kadaver), die als saprophagär wirken, aber auch nekrotische Gewebe in einem lebenden Wirt beeinflussen können. Die häufigsten Gattungen sind: Luca, Phaenicia, Musca und Fannia. Pseudomyiasis (zufällig): Dies kann durch Die Einnahme von Ei- und/oder Dipteranlarven auftreten, die in kontaminierten Lebensmitteln enthalten sind, die vom Wirt aufgenommen werden und eine Darmerkrankung verursachen (CAVALCANTI, 2008) (BOROS; BOROS et al., 2006).

\section{KLINISCHER FALL}

Ein 69-jähriger männlicher Patient, bei dem ein bösartiges Neoplasma in der Zunge diagnostiziert wurde (chemotherapiend), wurde in die Notaufnahme des Dom Moura Regional hospital in Garanhuns eingeliefert - Pernambuco klagte mehrere Wochen über Schmerzen und Seltenheit im Mundbereich, wurde an den Bucomaxillofacial Surgery and Traumatology Service verwiesen. Bei der Aufnahme war der Patient afebrile, orientiert und kooperativ, Blutungen wurden im Mund beobachtet, unter Berücksichtigung der Familie Bericht, dass der Patient Zahnprobleme hatte, und Untersuchungen wurden durchgeführt. In der intraoralen Auswertung zeigte eine klar definierte ulzerative Läsion mit zentralem nekrotischem Gewebe und mehreren lebenden Larven auf der Zunge. (Abbildung 1)

RC: 61098

Disponível

em: https://www.nucleodoconhecimento.com.br/gesundheit/humanmyiasis 
Abbildung 1 - Larven auf der Zunge.

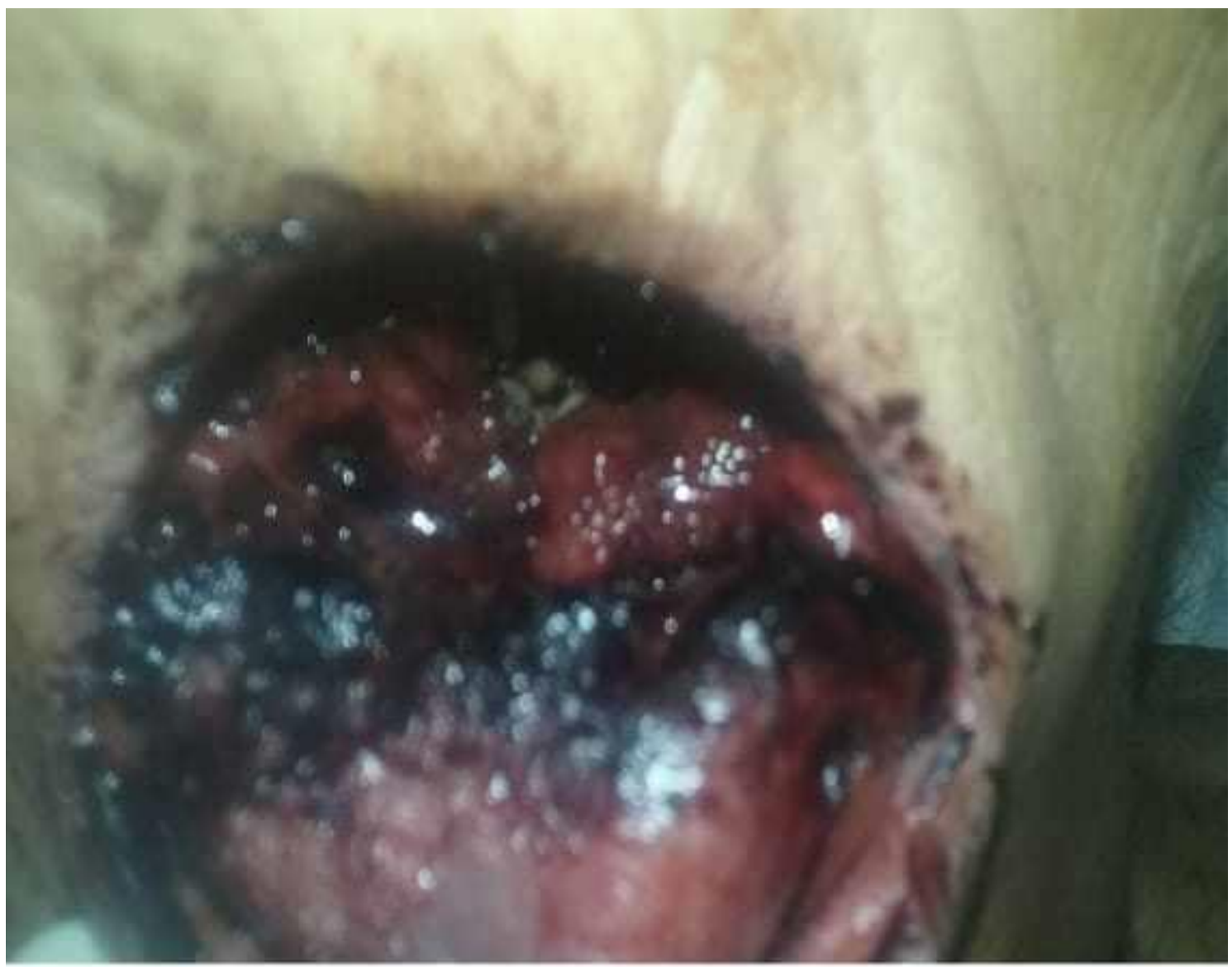

Quelle: Die Autoren (2020).

Der von der Krankheit betroffene Patient präsentierte prädisponierende Faktoren wie körperliche und geistige Schwäche, Dehydrierung, unzureichende Körperhygiene, Diabetes, Unterernährung, Mundatmung während des Schlafes und schlechte Erhaltung der Mundhygiene.

So wurde eine primäre symptomatische Behandlung durchgeführt. Die Wunde wurde mit 0,12\% Chlorhexidin-Lösung gereinigt und desinfiziert. Die Larven wurden manuell unter örtlicher Anästhesie mit Hilfe einer klinischen Pinzette entfernt, das gesamte aseptische Feld, die Larven in diesem Fall wurden gesammelt und in ein klinisches Tablett gelegt. Etwa 80 Larven wurden aus der Region entfernt, und alle konnten nicht entfernt werden (Abbildung 2).

RC: 61098

Disponível

em: https://www.nucleodoconhecimento.com.br/gesundheit/humanmyiasis 
Abbildung 2 - Aus der Läsion entfernte Larven

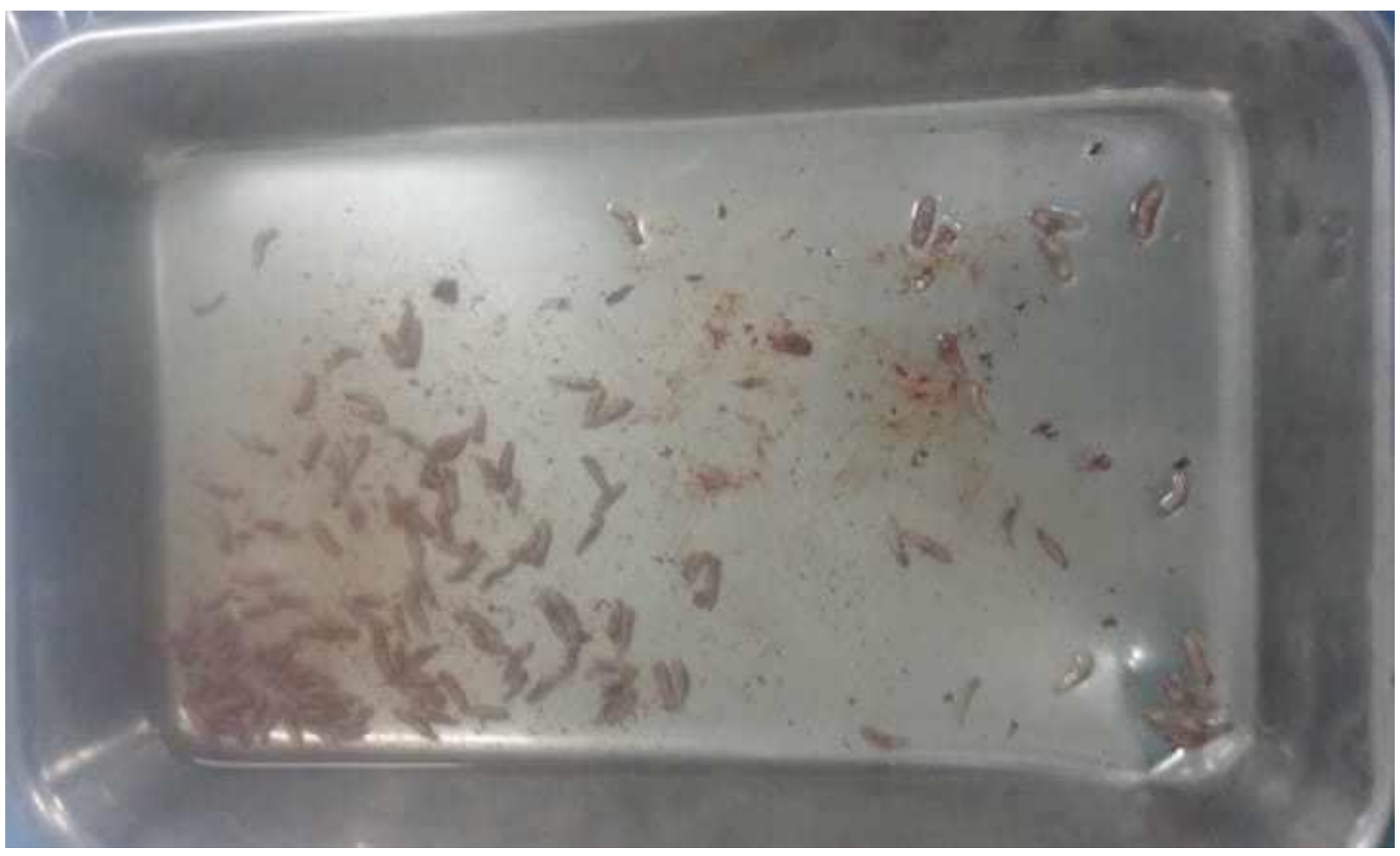

Quelle: Die Autoren (2020).

Der Patient wurde ins Krankenhaus eingeliefert und intravenöse Antibiotikatherapie wurde cephalotin 1g, Dipirona und Ketoprofen begonnen, und eine einzige orale Dosis von Ivermectin zwei Tabletten, d.h. 12 mg (ca. 200 g/kg), Nach 10 Tagen waren alle verbleibenden Larven ausgerottet worden, ohne dass eine Ergänzung von Ivermectin erforderlich war.

\section{DISKUSSION}

Während des Prozesses der Entwicklung von Larven, benachbarte Gewebe präsentieren entzündlichen Prozess, mit oder ohne Ulzeration oder Nekrose des Gewebes (CARVALHO; SANTOS et al., 2008). Viele schwerwiegende Komplikationen können durch Myiasis auftreten: Cellulitis, Erosion von Knochen und Zähnen, Bakteriämie und Tod (SZAKACS und MACPHERSON, 2007). 
In vielen der konsultierten Artikel haben wir beobachtet, dass die Autoren erwähnen, dass orale Myiasis eine seltene Pathologie ist und dass Dipteraner keine Vorliebe für Alter, Geschlecht und ethnische Zugehörigkeit haben. Und dass der Befall der Larven von Dipteranen eher auf dem Land ist, Haustiere wie Hunde, Katzen, Schweine, Ochsen befallen. Die allgemeine Meinung der Autoren ist, dass Menschen, die von Larven befallen wurden, geistig behindert sind, Menschen mit wenig Körper- und Mundhygiene, wie z. B. niedriges Bildungsniveau, Menschen mit niedrigem sozioökonomischen Status (BOROS; BOROS et al., 2006).

Die Diagnose der menschlichen Myiasis ist klinisch, so dass man einige seiner Komplikationen erkennen kann, um Computertomographie, Ultraschall oder Magnetresonanztomographie durchzuführen (SHENOUDA; ENTEN et al., 2018).

Das Vorhandensein von Larven auf der Haut löst eine lokale Entzündungsreaktion mit der Migration und Proliferation von Entzündungszellen wie Neutrophilen, Mastzellen, Eosinophilen, Fibroblasten und Endothelzellen aus. Die vollständige Anzahl der Blutkörperchen kann jedoch einen hohen Gehalt an Leukozyten und Eosinophilen aufweisen (PAYNE und COSGROVE, 1966) (SHENOUDA; ENTEN et al., 2018).

Einige Autoren befürworten eine konventionelle Behandlung von Myiasis, die die Entfernung von Larven mit Hilfe chemischer Substanzen umfasst, die darauf abzielt, die Asphyxie von Larven zu fördern, die ihren Ausstieg aus der Läsion verursachen. Bei größeren Läsionen, wo es ein klinisches Bild der benachbarten Zellulitis präsentiert, würde eine Debridement von lokalen Geweben mit der Entfernung von nekrotischen Geweben angezeigt werden, aber andere verteidigen als ideal, die chirurgische Behandlung, die die Entfernung aller Larven mit einem Debridement verbunden ermöglicht, und Antibiotika, um sekundäre Infektion zu verhindern, eine andere Methode wurden bereits als die Verwendung einer einzigen Dosis von Ivermectin (PARMAR; DAVESSAR et al., 2018).

RC: 61098

Disponível

em: https://www.nucleodoconhecimento.com.br/gesundheit/humanmyiasis 
Myiasis kann als ein Problem der öffentlichen Gesundheit angesehen werden, das durch die Beseitigung von Risikofaktoren für die menschliche Gesundheit verhindert werden kann, wie z. B. fehlende sanitäre Grundversorgung und unzureichende Entsorgung von Müll, wo organisches Material freigelegt wird, das Insekten und Kleintiere anzieht und einen nachhaltigen Kreislauf von Schmutz schafft. Schlechte sanitäre Einrichtungen sind wahrscheinlich der wichtigste Risikofaktor für humane Myiasis (FRANCESCONI und LUPI, 2012).

\section{FAZIT}

In der Literatur wurden mehrere Behandlungen für Kavitary Myiasis vorgeschlagen, von der mechanischen Extraktion bis zur Verwendung von topischen, oralen und intravenösen Substanzen. Aber eine frühe und korrekte Diagnose ermöglicht eine Behandlung mit einer günstigen Prognose. Unabhängig von den eingesetzten Mitteln ist es jedoch wichtig, dass sich der Patient an der Annahme geeigneter persönlicher Hygienemaßnahmen orientiert. Es gibt jedoch keinen Konsens über den besten therapeutischen Ansatz für Fälle von oraler Myiasis.

\section{VERWEISE}

BARNABÉ, A. S. et al. Epidemiologia Da Miíase Cutânea: Revisão Da Literatura. Atas de Ciências da Saúde, São Paulo, v. 4, n. 2, p. 14-22, Abril - Junho 2016.

BOROS, L. F. et al. Míase Labial. Revista Dens, v. 14, n. 1, p. 1-10, Revista Dens, v.14, n.1, maio/outubro 20062006.

CARVALHO, R. W. F. et al. Oral and maxillofacial myiasis associated with epidermoid carcinoma: a case report. Journal of Oral Science, v. 50, n. 1, p. 103-105, 2008.

CAVALCANTI, A. L. Miíase Oral: etiologia, diagnóstico e tratamento. Rev. Fac. Odontol. Porto Alegre, Porto Alegre, v. 49, n. 2, p. 32-35, maio/ago. 2008.

RC: 61098

Disponível

em: https://www.nucleodoconhecimento.com.br/gesundheit/humanmyiasis 
CAVALCANTI, A. L. Miíase Oral: etiologia, diagnóstico e tratamento. Rev. Fac. Odontol. Porto Alegre, Porto Alegre, v. 46, n. 2, p. 32-35, maio/ago. 2008.

FRANCESCONI, ; LUPI,. Myiasis. Clinical Microbiology Reviews , v. 25, n. 1, p. 79 105, Jan. 2012.

GEALH, W. C. et al. Treatment of oral myiasis caused by Cochliomyia hominivorax: two cases treated with ivermectin. British journal of Oral and Maxillofacial Surgeons, v. 47 , n. 1, p. 23-26, January 2009.

GUIMARAES, J. H.; PAPAVERO, ; PRADO, A. P. D. As Mi1ases Na Região Neotropical. Revista Brasileira De Zoologia (Identificação, Biologia, Bibliografia), São Paulo, v. 1, n. 4, p. 239.416, 1983.

KHAN, B. A. et al. Oral and cutaneous myiasis in a 5 year old child from Karachi, Pakistan. Le Infezioni in Medicina, v. 4, p. 385-388, 2018.

MARQUEZ, A. T.; MATTOS, M. D. S.; NASCIMENTO, S. B. Míases associadas com alguns fatores sócio-econômicos em cinco áreas urbanas do Estado do Rio de Janeiro. Revista da Sociedade Brasileira de Medicina Tropical, v. 40, n. 2, p. 175180, março - abril 2007.

NASCIMENTO, E. M. F. D. et al. Miíases Humanas Por Cochliomyia Hominivorax (Coquerel, 1858) (Diptera, Calliphoridae) Em Hospitais Públicos Na Cidade Do Recife, Pernambuco, Brasil. Entomología y Vectores, v. 12, n. 1, p. 37-51, 2005.

PARMAR, S. et al. Tracheostomal Myiasis: A Case Report And Review Of Literature. International Journal of Scientific Research, v. 7, n. 12, p. 4-6, December 2018.

PAYNE, J. A.; COSGROVE, G. E. Tissue changes following Cuterebra infestation in rodents. The American Midland Naturalist, v. 75, n. 1, p. 205-213, Jan. 1966.

RC: 61098

Disponível

em: https://www.nucleodoconhecimento.com.br/gesundheit/humanmyiasis 
RIBEIRO, F. A. Q. et al. Tratamento da miíase humana cavitária com ivermectina oral. Rev Bras Otorrinolaringol, v. 67, n. 6, p. 755-761, nov./dez. 2001.

SHENOUDA, M. et al. Human Botfly: A Case Report and Overview of Differential Diagnosis. J Investig Med High Impact Case Rep., v. 6, Jan-Dec; 2018.

SILVEIRA, M. A. A. et al. Cavitary myiasis mimicking peritonsilar abscess. Brazilian Journal of Otorhinolaryngology, v. 81, p. 336-338, 2015.

SZAKACS, T. A.; MACPHERSON,. Nosocomial myiasis in a Canadian intensive care unit. CMAJ, v. 177, n. 7, p. 719-720, 25 September 2007.

TOURÉ, S. M. Les myiases d'importance économique. Rev. sci. tech. Off. int. Epiz., 1994, 13 (4), 1053-1073, v. 13, n. 4, p. 1053-1073, 1994.

VINIT, G. B. ; JAYAVELU, P.; SHRUTHA, S. P. Oral myiasis in a maxillofacial trauma patient. J Pharm Bioallied Sci., v. 5, n. 6, p. 195-197, 5 Jul 2013.

Eingesandt: Februar 2020.

Genehmigt: August 2020.

RC: 61098

Disponível

em: https://www.nucleodoconhecimento.com.br/gesundheit/humanmyiasis 